\title{
Impacto da estocagem sobre atividade antioxidante e teor de ácido ascórbico em sucos e refrescos de tangerina
}

\author{
Storage impact on the antioxidant activity \\ and ascorbic acid level of mandarin \\ juices and drinks
}

Carolina Ferraz Figueiredo MOREIRA ${ }^{1}$

Maria Lúcia Mendes LOPES ${ }^{1}$

Vera Lúcia VALENTE-MESQUITA ${ }^{1}$

\section{RE S U M O}

\section{Objetivo}

Objetivou-se avaliar a estabilidade do ácido ascórbico em suco in natura de tangerina cultivar Ponkan sob diferentes condições de armazenamento, e em 13 amostras de bebidas industrializadas de tangerina, bem como a estabilidade da atividade antioxidante no suco in natura.

\section{Métodos}

O suco in natura de tangerina foi armazenado em três temperaturas: ambiente, refrigeração e congelamento. Amostras de tangerinas foram armazenadas sob refrigeração. Treze marcas de bebidas industrializadas de tangerina foram adquiridas e mantidas sob refrigeração. Foram determinados o teor de ácido ascórbico pelo método titulométrico de Tillmans (Association of Official Analytical Chemists) e a atividade antioxidante por meio da capacidade sequestradora do radical 2,2-difenil-1-picril-hidrazila.

\section{Resultados}

O suco in natura apresentou teor médio de ácido ascórbico de 32,40mg/100mL; a taxa de redução deste nutriente foi maior em temperatura de estocagem mais elevada. A atividade antioxidante média foi de 89,74\% de inibição do radical 2,2-difenil-1-picril-hidrazila, apresentando variações de até 4,26\% durante o armazenamento. Nas bebidas industrializadas, o teor médio de ácido ascórbico variou entre 1,01 e 10,72mg/100mL, com perdas de até $82,76 \%$. Com base na legislação brasileira, seis marcas de bebidas industrializadas apresentaram não conformidades em relação à declaração do teor de ácido ascórbico nos rótulos.

\footnotetext{
${ }^{1}$ Universidade Federal do Rio de Janeiro, Instituto de Nutrição Josué de Castro, Departamento de Nutrição Básica e Experimental. Av. Carlos Chagas Filho, 373, Bloco J, $2^{\circ}$ andar, Sala 16, 21941-902, Rio de Janeiro, RJ, Brasil. Correspondência para/Correspondence to: VL VALENTE-MESQUITA. E-mail: <valentem@nutricao.ufrj.br>.
} 
744 | CFF MOREIRA et al.

\section{Conclusão}

O suco de tangerina cultivar Ponkan apresenta elevada atividade antioxidante, inclusive durante o armazenamento. Observou-se superioridade nutricional do suco fresco em comparação às bebidas industrializadas, considerando o teor de ácido ascórbico e sua estabilidade. Adicionalmente, os dados obtidos apontam para a necessidade de maior fiscalização em relação à rotulagem nutricional.

Termos de indexação: Ácido ascórbico. Antioxidante. Armazenamento de alimento. Citrus.

\section{A B S T R A C T}

\section{Objective}

The objective of this study was to analyze the ascorbic acid stability of mandarin juice from the cultivar Ponkan under different storage conditions, and of 13 samples of processed mandarin beverages, as well as the antioxidant activity stability of fresh juice.

\section{Methods}

Fresh mandarin juice was stored under three temperatures: room temperature, refrigeration and freezing. Mandarin samples were kept under refrigeration. Thirteen brands of processed mandarin beverages were purchased and kept under refrigeration. Ascorbic acid level was determined by the Tillman's titrimetric method (Association of Official Analytical Chemists) and antioxidant activity by the radical scavenger capacity using the 2,2-diphenyl-1-picrylhydrazyl radical.

\section{Results}

The mean ascorbic acid level in fresh juice was $32.40 \mathrm{mg} / 100 \mathrm{~mL}$ and the reduction rate of this nutrient was higher when the samples were stored under higher temperatures. The average antioxidant activity was $89.74 \%$ of 2,2-diphenyl-1-picrylhydrazyl radical inhibition, with variations of up to $4.26 \%$ during storage. Among the processed beverages, the mean ascorbic acid content varied from 1.01 to $10.72 \mathrm{mg} / 100 \mathrm{~mL}$, with losses of up to $82.76 \%$. Six brands of processed beverages were considered noncompliant with the Brazilian legislation regarding the ascorbic acid content reported in the labels.

\section{Conclusion}

Mandarin juice from the Ponkan cultivar has high antioxidant activity, even during storage. The fresh juice was nutritionally better than the processed beverages, considering ascorbic acid content and stability. Additionally, the results indicated the need of greater surveillance of nutritional labeling.

Indexing terms: Ascorbic acid. Antioxidants. Food storage. Citrus

\section{N T R O D U ÇÃ O}

Os antioxidantes estão presentes em frutas e hortaliças e têm importante papel na redução do risco de ocorrência de doenças crônicas não transmissíveis, como as cardiovasculares e neurológicas, diabetes Mellitus, câncer e obesidade, por promover proteção contra a presença de radicais livres no organismo. Atualmente, observa-se o aumento da incidência dessas doenças, o que pode estar associado a mudanças de hábitos alimentares ${ }^{1,2}$. As frutas cítricas são conhecidas por serem fontes de compostos antioxidantes, como, por exemplo, Ácido Ascórbico (AA), compostos fenólicos e carotenoides ${ }^{3}$.
As tangerinas (Citrus reticulata Blanco) constituem o segundo grupo de frutas cítricas mais importantes na citricultura mundial, sendo o Brasil o quarto maior produtor ${ }^{4}$. Uma das principais características dessa fruta é o elevado teor de $A A$, uma das formas ativas de vitamina $C$. $O$ AA não é sintetizado pelo organismo humano e está presente, principalmente, em frutas cítricas e hortaliças folhosas ${ }^{5}$. Comparada a outros nutrientes, essa vitamina é mais sensível à degradação durante o processamento e a estocagem, podendo, portanto, ser utilizada como indicador da qualidade nutricional de produtos derivados de frutas e hortaliças ${ }^{6}$. 
As tangerinas agradam ao paladar e são de fácil consumo: no Brasil, 58\% da tangerina comercializada correspondem ao cultivar Ponkan. Entre 2005 e 2010, a produção de tangerinas manteve-se estável. Esse cultivar é originário da Índia e apresenta coloração laranja acentuada, sabor doce, casca pouco aderida aos gomos e maior tamanho, quando comparado aos demais cultivares $^{4,7-9}$.

Com o ritmo de vida acelerado, principalmente nos grandes centros urbanos, é crescente a demanda por produtos industrializados que sejam seguros e funcionais, o que contribui para o aumento do consumo de sucos e refrescos industrializados de frutas. De acordo com a Associação Brasileira das Indústrias de Refrigerantes e de Bebidas não Alcoólicas, em 2010, o consumo nacional per capita de bebidas para preparo e de bebidas prontas para beber foi de 30,1 e 43,7L, respectivamente. Entre 2005 e 2010, o consumo de sucos concentrados, de preparados sólidos para refresco e de sucos e néctares prontos para o consumo aumentaram $0,5,1,9$, e 10,6\%, respectivamente. Os sucos concentrados, normalmente comercializados sob a forma congelada, e os preparados sólidos para refresco são facilmente elaborados e, assim como os sucos prontos para o consumo, podem ser estocados e têm boa aceitação entre os consumidores de todas as idades $^{10-12}$.

Embora a tangerina seja produzida em grande quantidade no Brasil, e o consumo de bebidas industrializadas venha crescendo nos últimos anos, são escassos estudos sobre os produtos obtidos a partir dessa fruta. Este trabalho teve como objetivo avaliar a estabilidade do ácido ascórbico em suco in natura de tangerina cultivar Ponkan, sob diferentes condições de armazenamento, e em suco de frutas que permaneceram inteiras e estocadas sob refrigeração, bem como em bebidas industrializadas sabor tangerina. A estabilidade da atividade antioxidante do suco in natura também foi determinada.

\section{MÉ T O D O S}

As tangerinas e as bebidas industrializadas foram selecionadas por amostragem não probabilística a esmo ${ }^{13}$. Foram adquiridos $36 \mathrm{~kg}$ de tangerinas cultivar Ponkan, em estágio de maturação comercial, em um fornecedor atacadista da cidade do Rio de Janeiro (Brasil).

As frutas foram divididas em dois lotes. Em um deles, o suco das frutas foi obtido em extrator (Samsom, EUA) e subdividido em três alíquotas, que foram mantidas nas seguintes condições: em temperatura ambiente (Média - $M=25$, Desvio-Padrão-DP $=3^{\circ} \mathrm{C}$ ) por 8 horas, sob refrigeração $\left(M=4, D P=3^{\circ} \mathrm{C}\right)$ por quatro dias e sob congelamento $\left(\mathrm{M}=-22, \mathrm{DP}=1^{\circ} \mathrm{C}\right)$ por 120 dias. Amostras de cada uma dessas alíquotas foram coletadas para análise em diferentes intervalos de tempo: a cada 2 horas, diariamente e a cada 30 dias, respectivamente. No outro lote, as tangerinas foram armazenadas sob refrigeração por 28 dias, e, semanalmente, 20 unidades de frutas desse lote tiveram o suco extraído e analisado.

Foram adquiridos em mercados varejistas do estado do Rio de Janeiro, três lotes de 13 diferentes marcas de bebidas industrializadas, sendo 11 de Preparados Sólidos para Refresco (PSR), sabor tangerina, uma de Suco de Pronto para o Consumo (SPC) de tangerina e uma de suco de tangerina concentrado congelado, totalizando 39 amostras. A amostra correspondente a cada lote de bebidas industrializadas foi composta pelo conteúdo homogeneizado de três embalagens. As amostras de SPC foram armazenadas em sua própria embalagem e mantidas sob refrigeração $\left(\mathrm{M}=4, \mathrm{DP}=3^{\circ} \mathrm{C}\right)$ por aproximadamente 60 horas. Os PSR e as amostras de suco concentrado congelado foram diluídos com água filtrada na proporção recomendada pelo fabricante, conforme especificado nas embalagens dos produtos, acondicionados em recipientes plásticos opacos, tampados e mantidos sob refrigeração pelo mesmo período.

Imediatamente após a extração do suco in natura e da obtenção das bebidas industrializadas, foram determinados em triplicata: Acidez 
Total Titulável (ATT), por titulação com NaOH 0,1 $\mathrm{N}$; $\mathrm{pH}$ em potenciômetro digital (Micronal, Brasil); e teor de Sólidos Solúveis Totais (SST) em refratômetro ${ }^{14}$ (Atago, Japão). Logo após a obtenção do suco in natura e das bebidas industrializadas, e, periodicamente, durante a estocagem, foi determinado o teor de $\mathrm{AA}$, em triplicata, pelo método titulométrico de Tillmans (Association of Official Analytical Chemists) ${ }^{15}$, modificado por Benassi \& Antunes ${ }^{16}$, que utilizaram solução de ácido oxálico como solvente, em substituição ao ácido metafosfórico. A uma alíquota de $20 \mathrm{~mL}$ de amostra foram adicionados $80 \mathrm{~mL}$ de ácido oxálico $1 \%$ e $10 \mathrm{~mL}$ dessa solução foram titulados com reagente de Tillmans, preparado com a utilização de 2,6 diclorofenolindofenol. Os resultados foram calculados com base em uma solução padrão de AA e expressos em mg de AA por $100 \mathrm{~mL}$ de suco ou de refresco. Com base nos padrões estabelecidos pela Resolução RDC $n^{\circ} 360$ de $2003^{17}$, foi avaliada a adequação dos teores de AA declarados nos rótulos em relação aos obtidos a partir da análise das bebidas industrializadas.

Paralelamente, para os mesmos períodos de estocagem, determinou-se a atividade antioxidante do suco de tangerina in natura, em duplicata, por meio da capacidade sequestradora do radical 2,2-Difenil-1-Picril-Hidrazila (DPPH) por compostos antioxidantes presentes na matriz alimentar, conforme descrito por Kuskoski et al. ${ }^{18}$. Em ausência de luz, uma alíquota de $100 \mu \mathrm{L}$ de amostra foi adicionada a $3,9 \mathrm{~mL}$ de solução de DPPH $100 \mu \mathrm{M}$, sendo a mistura incubada em temperatura ambiente por 60 minutos. A absorbância foi lida a $517 \mathrm{~nm}$ e a atividade foi calculada como porcentagem de inibição do radical DPPH conforme fórmula \% inibição radical DPPH $=\left(A_{0}-A_{t} / A_{0}\right) x$ 100, onde $A_{0}$ representa a absorbância da solução de DPPH medida no tempo zero, e $\mathrm{A}_{\mathrm{t}^{\prime}}$ a absorbância após 60 minutos de reação da solução de DPPH com a amostra. O valor de $A_{0}$ é considerado $100 \%$.

Os dados de teor de ácido ascórbico das bebidas industrializadas foram submetidos à Aná- lise de Variância (Anova) e as médias comparadas pelo teste de Tukey $(p<0,05)$. Os resultados obtidos ao longo do período de estocagem de todas as amostras foram submetidos à análise de regressão linear simples. Foi utilizado o software estatístico Microsoft ${ }^{\circledR}$ Excel 2000 (Microsoft Corporation, Redmond, USA).

\section{RESULTADOS}

O suco de tangerina cultivar Ponkan apresentou os seguintes valores médios para os parâmetros físico-químicos: ATT, 0,50g\% de ácido cítriCO, pH, 4,07, SST, 9,5 Brix e Ratio (SST/ATT), 19. O teor médio de AA do suco foi de $32,40 \mathrm{mg} /$ $100 \mathrm{~mL}$. Dessa forma, o consumo de $200 \mathrm{~mL}$ (1 copo) de suco de tangerina cultivar Ponkan ultrapassa a ingestão dietética de referência (IDR) de AA para adultos ${ }^{19}$, sendo considerado fonte desse nutriente.

A Figura 1 apresenta a estabilidade do AA em suco de tangerina cultivar Ponkan em diferentes condições de estocagem. A redução no teor médio dessa vitamina foi de, no máximo, 8,05\% no suco das frutas que permaneceram inteiras e estocadas sob refrigeração.

Durante o período de estocagem, a taxa de redução do AA foi de 2,869; 0,380; e 0,008mg/dia para os sucos armazenados em temperatura ambiente, sob refrigeração e sob congelamento, respectivamente. Observou-se que quanto maior a temperatura de estocagem, maior a taxa de redução de AA. Para o suco extraído das tangerinas armazenadas inteiras sob refrigeração, a taxa de redução de AA foi de $0,038 \mathrm{mg} /$ dia. Essa taxa corresponde a $10 \%$ daquela obtida para o suco armazenado nas mesmas condições.

A atividade antioxidante média do suco de tangerina cultivar Ponkan imediatamente após sua extração foi de $89,74 \%$ de inibição do radical DPPH. Ao longo do período de armazenamento, foi observada variação de 4,26\% na atividade antioxidante do suco estocado sob congelamento (Figura 2). 
(a)

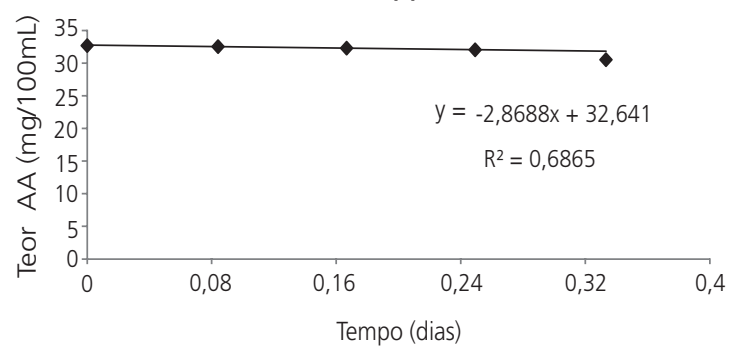

(c)

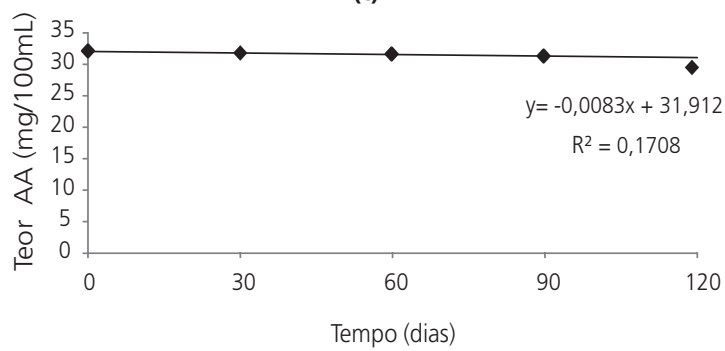

(b)

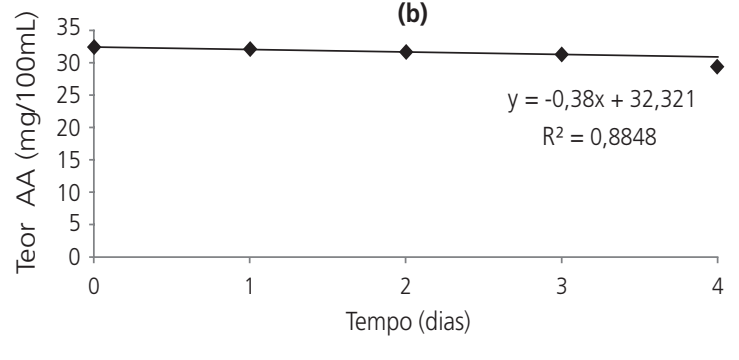

(d)

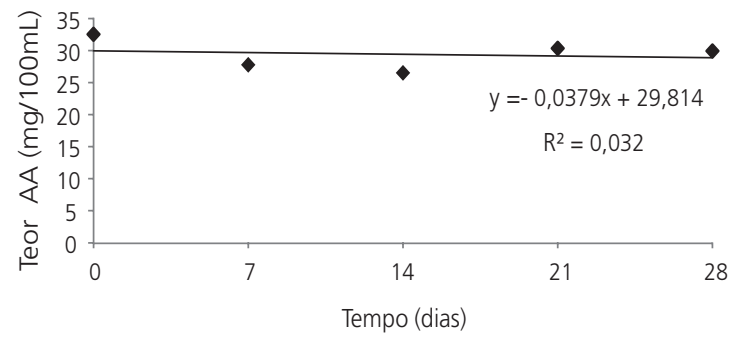

Figura 1. Estabilidade do Ácido Ascórbico (AA) em suco de tangerina cultivar Ponkan mantido em diferentes condições de estocagem: (a) temperatura ambiente $\left(M=25, D P=3^{\circ} \mathrm{C}\right)$; (b) refrigeração $\left(M=4, D P=3^{\circ} \mathrm{C}\right)$; (c) congelamento $\left(M=-22, D P=1^{\circ} \mathrm{C}\right)$; e (d) tangerinas mantidas inteiras sob refrigeração $\left(M=4, D P=3^{\circ} \mathrm{C}\right)$. Rio de Janeiro (RJ), 2009.

Nota: M: Média; DP: Desvio-Padrão.

(a)

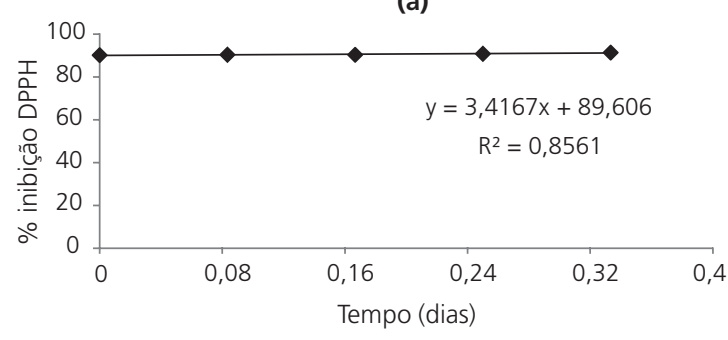

(c)

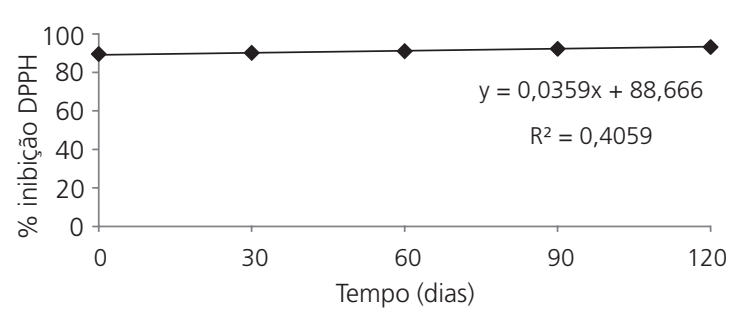

(b)

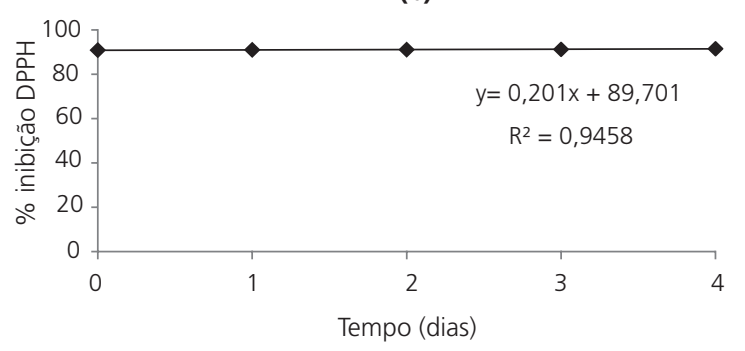

(d)

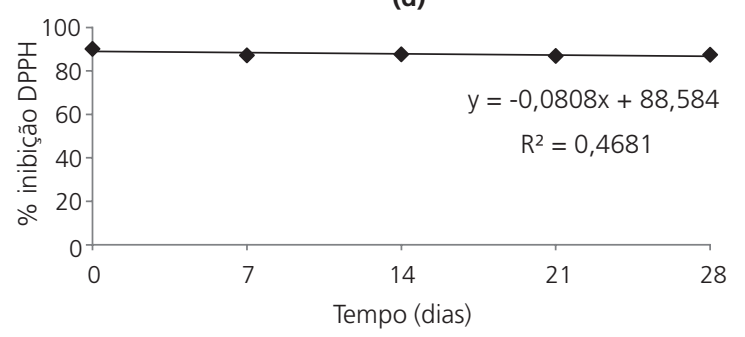

Figura 2. Atividade antioxidante de suco de tangerina cultivar Ponkan mantido em diferentes condições de estocagem: (a) temperatura ambiente $\left(M=25, D P=3^{\circ} \mathrm{C}\right)$; (b) refrigeração $\left(M=4, D P=3^{\circ} \mathrm{C}\right) ;(c)$ congelamento $\left(M=-22, D P=1^{\circ} \mathrm{C}\right)$; e (d) tangerinas inteiras mantidas sob refrigeração $\left(M=4, D P=3^{\circ} \mathrm{C}\right)$. Rio de Janeiro (RJ), 2009.

Nota: M: Média; DP: Desvio-Padrão. 
Os valores médios de ATT, de $\mathrm{pH}$ e, principalmente, de SST variaram entre as amostras de bebidas industrializadas, sendo essa variação entre 0,21 e 0,85g\% de ácido cítrico, 2,44 e 3,52 e 1,00 e $10,47^{\circ}$ Brix, respectivamente (Tabela 1 ).
Como esperado, os maiores valores obtidos para esses parâmetros correspondem à única amostra de SPC (amostra 12).

A Tabela 2 apresenta o teor médio de AA, a taxa de redução e a porcentagem de perda de

Tabela 1. Valores médios de ATT, pH e STT em bebidas industrializadas de tangerina. Rio de Janeiro (RJ), 2009.

\begin{tabular}{|c|c|c|c|c|c|c|}
\hline \multirow{2}{*}{ Amostras } & \multicolumn{2}{|c|}{$\mathrm{ATT}^{*}$ ( $\mathrm{g} \%$ ácido cítrico) } & \multicolumn{2}{|c|}{$\mathrm{pH}^{*}$} & \multicolumn{2}{|c|}{ SST ( ${ }^{\circ}$ Brix) ${ }^{*}$} \\
\hline & $M$ & $\mathrm{DP}$ & M & DP & $M$ & DP \\
\hline 1 & 0,45 & 0,01 & 0,45 & 0,01 & 1,00 & 0,00 \\
\hline 2 & 0,42 & 0,12 & 0,42 & 0,12 & 3,00 & 0,00 \\
\hline 3 & 0,65 & 0,02 & 0,65 & 0,02 & 3,50 & 0,00 \\
\hline 4 & 0,64 & 0,01 & 0,64 & 0,01 & 1,00 & 0,00 \\
\hline 5 & 0,85 & 0,13 & 0,85 & 0,13 & 1,00 & 0,17 \\
\hline 6 & 0,74 & 0,15 & 0,74 & 0,15 & 3,43 & 0,11 \\
\hline 7 & 0,44 & 0,04 & 0,44 & 0,04 & 4,37 & 0,11 \\
\hline 8 & 0,63 & 0,07 & 0,63 & 0,07 & 4,37 & 0,11 \\
\hline 9 & 0,44 & 0,07 & 0,44 & 0,07 & 3,83 & 0,29 \\
\hline 10 & 0,47 & 0,04 & 0,47 & 0,04 & 1,00 & 0,00 \\
\hline 11 & 0,58 & 0,04 & 0,58 & 0,04 & 1,00 & 0,00 \\
\hline 12 & 0,69 & 0,07 & 0,69 & 0,07 & 10,47 & 0,15 \\
\hline 13 & 0,21 & 0,04 & 0,21 & 0,04 & 6,87 & 1,33 \\
\hline
\end{tabular}

Amostras 1 a 11 - PSR: Preparados Sólidos para Refresco; Amostra 12 - SPC: Suco Pronto para o Consumo; e Amostra 13 bebida preparada a partir de suco concentrado congelado. ATT: Acidez Total Titulável; SST: Sólidos Solúveis Totais; M: Média; DP: Desvio-Padrão.

*As análises foram feitas em triplicata de três diferentes lotes, totalizando nove determinações.

Tabela 2. Teor médio de AA de bebidas industrializadas de tangerina, taxa de redução e porcentagem de perda de $A A$ durante a estocagem, diferença do teor de AA em relação ao rótulo e porcentagem da ingestão dietética de referência ${ }^{*}$ suprida pelo consumo de 200mL (1 copo). Rio de Janeiro (RJ), 2009.

\begin{tabular}{|c|c|c|c|c|c|c|}
\hline \multirow{2}{*}{ Amostras } & \multicolumn{2}{|c|}{ Teor de $\mathrm{AA}(\mathrm{mg} / 100 \mathrm{~mL})^{* *}$} & \multirow{2}{*}{$\begin{array}{c}\text { Taxa de redução } \\
\text { AA (mg/hora) }\end{array}$} & \multirow{2}{*}{$\%$ perda AA } & \multirow{2}{*}{$\begin{array}{c}\text { \% diferença para o } \\
\text { rótulo }\end{array}$} & \multirow{2}{*}{$\%$ IDR $(200 \mathrm{~mL})$} \\
\hline & M & DP & & & & \\
\hline 1 & $1,02^{g}$ & 0,00 & 0,0041 & 17,65 & Não consta & 4,53 \\
\hline 2 & $4,87^{\text {ef }}$ & 0,99 & 0,0496 & 82,76 & $+45,37$ & 21,64 \\
\hline 3 & $7,07^{\mathrm{cd}}$ & 1,42 & 0,0325 & 31,68 & $+5,73$ & 31,42 \\
\hline 4 & $1,67^{\mathrm{g}}$ & 0,66 & 0,0100 & 60,87 & Não consta & 7,42 \\
\hline 5 & $5,34^{\text {def }}$ & 2,20 & 0,0268 & 29,68 & $-33,25$ & 23,73 \\
\hline 6 & $8,09^{b c}$ & 1,48 & 0,0914 & 78,03 & $+19,85$ & 35,96 \\
\hline 7 & $6,96^{\text {cde }}$ & 0,31 & 0,0467 & 32,43 & $+16,00$ & 30,93 \\
\hline 8 & $7,60^{\text {bc }}$ & 1,00 & 0,0280 & 28,83 & $+1,33$ & 33,78 \\
\hline 9 & $7,09^{c d}$ & 1,24 & 0,0513 & 45,48 & $+57,56$ & 31,51 \\
\hline 10 & $9,36^{\mathrm{ab}}$ & 0,56 & 0,0346 & 26,26 & $+24,80$ & 41,60 \\
\hline 11 & $1,01^{\mathrm{g}}$ & 0,50 & 0,0000 & 0,00 & Não consta & 4,49 \\
\hline 12 & $10,72^{\mathrm{a}}$ & 0,83 & 0,0802 & 42,64 & Não consta & 47,64 \\
\hline 13 & $4,58^{f}$ & 0,30 & 0,0350 & 28,58 & $-8,4$ & 20,36 \\
\hline
\end{tabular}

Amostras 1 a 11 - PSR: Preparados Sólidos para Refresco; Amostra 12 - SPC: Suco Pronto para o Consumo; e Amostra 13 - bebida preparada a partir de suco concentrado congelado.

Médias seguidas de mesma letra, em uma mesma coluna, não diferem entre si pelo teste de Tukey $(p<0,05)$.

*Ingestão dietética de referência de Ácido Ascórbico (AA) (45mg) para adultos ${ }^{19} ;{ }^{* *}$ As análises foram feitas em triplicata de três diferentes lotes, totalizando nove determinações; ${ }^{* * *}$ Análise x 100/Rótulo; M: Média; DP: Desvio-Padrão. 
AA durante a estocagem das bebidas industrializadas prontas para o consumo, a diferença do teor de AA em relação ao rótulo e a porcentagem da IDR suprida pelo consumo de $200 \mathrm{~mL}$ (1 copo). Foi observada diferença significativa no teor médio de AA entre algumas amostras de PSR, que variou de 1,01 a 9,36mg em $100 \mathrm{~mL}$ de bebida. O SPC e a bebida preparada a partir de suco concentrado congelado apresentaram teores médios de AA que diferiram estatisticamente e foram, respectivamente, de 10,72 e 4,58mg/100mL de bebida.

Durante a estocagem das bebidas industrializadas sob refrigeração, foi observada taxa de redução no teor de AA entre 0,004 e 0,091 mg/hora, com exceção da amostra 11 (PSR), em que o teor manteve-se constante. Verificaram-se perdas no teor de $A A$ que variaram entre 17,65 e $82,76 \%$.

\section{I S C USS Ã O}

Xu et al. ${ }^{20}$, estudando suco de tangerina obtido do mesmo cultivar, encontraram valores médios de ATT, SST, Ratio e teor médio de AA de $1,29 \mathrm{~g} \%$ de ácido cítrico, $12^{\circ} \mathrm{Brix}, 9,33$ e 28,27mg/ $100 \mathrm{~mL}$, respectivamente. Damiani et al. ${ }^{21}$ observaram valores de SST, de $\mathrm{pH}$ e de teor médio de AA de $10,28^{\circ}$ Brix, 4,08 e $56,71 \mathrm{mg} / 100 \mathrm{~mL}$, respectivamente. As diferenças encontradas entre 0 presente estudo e os citados, provavelmente, se devam a variações influenciadas por fatores genéticos, safra, condições edafoclimáticas e práticas de cultivo 22 .

Lavinas et al. ${ }^{23}$ encontraram taxas de redução de AA de 7,39, 1,160, e 0,046mg/dia em suco de caju armazenado em temperatura ambiente, sob refrigeração e sob congelamento, respectivamente. Valente-Mesquita et al. ${ }^{24}$ observaram que o suco de laranjas cultivar Pera estocadas inteiras sob refrigeração apresentou taxa de redução de AA de, aproximadamente, 0,2mg/dia. Ao comparar as taxas de redução obtidas no presente estudo com as dos trabalhos citados, tanto para o armazenamento do suco quanto para estoca- gem de frutas inteiras sob refrigeração, verifica-se a maior estabilidade da tangerina cultivar Ponkan.

A atividade antioxidante média do suco in natura foi elevada, e resultados como esse embasam a busca dos consumidores por produtos naturais e funcionais, que têm elevado o consumo de sucos de frutas cítricas ${ }^{25}$, e demonstram que a ingestão de tangerina ou do seu suco pode contribuir para uma dieta rica em compostos antioxidantes.

Em estudo realizado na China com suco de tangerina cultivar Ponkan, foi observado 29,67\% de inibição do radical $\mathrm{DPPH}^{20}$. Assim como verificado para o AA, essas diferenças devem estar relacionadas a fatores genéticos, safra, condições edafoclimáticas e práticas de cultivo ${ }^{26}$.

Não foram encontrados estudos que avaliem a estabilidade da atividade antioxidante em suco de tangerina. Klimczak et al. ${ }^{27}$, ao estudarem duas diferentes marcas de suco de laranja estocadas em três diferentes temperaturas (18, 28 e $38^{\circ} \mathrm{C}$ ) por seis meses, observaram alterações significativas na capacidade antioxidante para ambos os sucos em todas as condições de armazenamento avaliadas.

Ao se compararem os resultados observados para o suco in natura de tangerina cultivar Ponkan, observou-se que os parâmetros físico-químicos encontram-se dentro da faixa de variação verificada para as bebidas industrializadas. Caleguer, Toffoli \& Benassii28, ao realizar estudo com PSR sabor laranja, verificaram valores de ATT e de pH que estão de acordo com os do presente estudo, entretanto os resultados para SST divergem. Silva et al. ${ }^{29}$ observaram valores semelhantes aos do presente estudo para bebidas industrializadas de laranja.

Dentre as bebidas industrializadas, a que apresentou o maior teor de AA foi o SPC, e, ao compará-lo com o suco in natura de tangerina, observou-se que este apresentou teor de AA aproximadamente três vezes maior. Esse resultado ratifica a superioridade nutricional do suco in 
natura em relação aos produtos industrializados. Entre as 11 amostras de PSR avaliadas, as seis que apresentaram maior teor de AA tiveram adição dessa substância em sua formulação declarada no rótulo do produto. O uso do AA como aditivo alimentar para prevenir a deterioração do alimento por mecanismos oxidativos é permitido pela legislação brasileira ${ }^{30,31}$. O SPC e a bebida preparada a partir de suco concentrado congelado não continham adição desse nutriente.

De acordo com a legislação brasileira, dos PSR analisados, seis podem ser considerados "ricos" em vitamina C e dois como "fontes" dessa vitamina, tendo em vista que atingem valores superiores a 7,5 e 15\% da Ingestão Dietética da Referência (IDR) em $100 \mathrm{~mL}$ de bebida, respectivamente. O SPC e a bebida preparada a partir de suco concentrado congelado podem ser considerados como "fonte" e "rico" em vitamina C, respectivamente ${ }^{19,32}$.

Não foram encontrados estudos sobre o teor e a estabilidade de AA em bebidas industrializadas sabor tangerina, mas alguns autores estudaram esses parâmetros em bebidas derivadas de outras frutas. Silva et al. ${ }^{29}$, estudando 11 marcas de PSR sabor laranja, assim como no presente estudo, observaram diferença significativa no teor de AA entre algumas amostras, que variou de 0,67 a $32 \mathrm{mg} / 100 \mathrm{~mL}$ de bebida. Assim, o consumo de $200 \mathrm{~mL}$ (1 copo) desses refrescos de laranja supriria de 2,98 a $142 \%$ da IDR de AA para adultos, enquanto no presente estudo essa variação foi de 4,49 a 41,60\%. Esses mesmos autores também avaliaram 10 amostras de SPC sabor laranja e observaram variação no teor de AA entre 17,25 e $62,40 \mathrm{mg} / 100 \mathrm{~mL}$. Um copo do suco que apresentou o maior teor de AA superaria a IDR para adultos, enquanto a bebida com menor teor supriria $76,67 \%$. Neste estudo, observou-se que o SPC de tangerina supriria $47,64 \%{ }^{19}$.

Pereira et al. ${ }^{33}$, estudando oito marcas de bebidas preparadas a partir de sucos concentrados de caju, observaram teores de AA entre 7,56 e $15,20 \mathrm{mg} / 100 \mathrm{~mL}$ de suco, correspondendo a 33,60 e $67,56 \%$ da IDR de AA para adultos em $200 \mathrm{~mL}$, enquanto, no presente estudo, a bebida preparada a partir de suco concentrado congelado sabor tangerina supriria $20,36 \%{ }^{19}$.

Três entre as onze marcas de PSR analisadas não apresentavam, entre as informações nutricionais do rótulo, o teor de vitamina $C$ do produto, o que pode não configurar uma não conformidade, uma vez que a RDC $n^{\circ} 360$ de $2003^{17}$ estabelece não haver necessidade de informar o teor de uma vitamina quando ele for inferior a 5,0\% da IDR por porção indicada no rótulo. No entanto, a amostra 4 (PSR), por conter $7,42 \%$ da IDR de vitamina C em 200mL (1 copo), apresenta-se não conforme. Na análise dos rótulos do SPC e da bebida preparada a partir de suco concentrado congelado, verificou-se que o SPC não estava em conformidade com essa legislação, pois não há declaração do teor de vitamina $\mathrm{C}$ na embalagem, apesar de as análises demonstrarem que o consumo de um copo da bebida atingiria $47,64 \%$ da IDR desse nutriente para adultos.

A RDC $n^{\circ} 360$ de $2003^{17}$ também permite o limite de tolerância de $20,00 \%$ para mais ou para menos com relação aos valores de nutrientes de-clarados no rótulo. Ao comparar o teor de AA obtido analiticamente com a informação nutricional contida nas embalagens, foi observado que quatro marcas não estavam em conformidade com a legislação, pois os teores apresentados nos rótulos superam o limite de tolerância, atingindo até 57,56\% (amostra 9).

Caleguer, Toffoli \& Benassi ${ }^{28}$ avaliaram o teor de AA em 12 amostras de PSR sabor laranja e observaram que, na maioria, o teor declarado nos rótulos foi próximo ou inferior ao obtido analiticamente. Um estudo realizado com dez marcas de SPC de laranja demonstrou que seis delas apresentavam teor de AA diferente do especificado no rótulo, e, nas demais, não havia indicação do teor desse nutriente no produto ${ }^{29}$.

Durante o período de estocagem, foi observada perda máxima de 8,05\% no teor médio de AA para o suco in natura de tangerina cultivar Ponkan. Entre as bebidas industrializadas, esse percentual atingiu valor, aproximadamente, dez vezes maior $(82,76 \%)$, demonstrando maior estabilidade do suco in natura. 
Pereira et al. ${ }^{33}$ observaram que, entre diferentes marcas de SPC de caju, o teor de AA em uma delas manteve-se estável e, nas demais, houve redução entre 2,1 e 6,4\%. Para bebidas preparadas a partir de sucos concentrados, esses autores relataram perdas entre 0,5 e $8,8 \%$ no teor dessa vitamina, enquanto em três sucos, manteve-se estável. Os resultados observados para as bebidas industrializadas sabor caju demonstram maior estabilidade quando comparados aos de tangerina. Silva et al..$^{29}$ encontraram taxas de redução de AA semelhantes ao do presente estudo para PSR e inferiores para SPC, ambos de laranja, também armazenados sob refrigeração.

Este estudo demonstrou que o suco in natura de tangerina cultivar Ponkan apresentou elevada atividade antioxidante, inclusive durante o armazenamento. O consumo de $140 \mathrm{~mL}$ desse suco seria suficiente para atender a ingestão dietética de referência de ácido ascórbico para adultos, podendo esse produto ser considerado fonte desse nutriente. A taxa de redução de ácido ascórbico do suco de tangerina in natura mantido em temperatura ambiente foi maior quando comparada à dos sucos refrigerado e congelado. Observou-se, também, que o teor de ácido ascórbico e sua estabilidade foram maiores no suco fresco em comparação às bebidas industrializadas. Esses resultados reforçam o conceito de que uma alimentação rica em frutas contribui para o consumo de compostos antioxidantes, em contraposição aos produtos industrializados, tendo em vista os efeitos negativos que o processamento pode exercer sobre o valor nutricional dos alimentos.

\section{A GRADECIMENTOS}

Os autores agradecem à Fundação de Amparo à Pesquisa Carlos Chagas Filho do Estado do Rio de Janeiro (E-26/110.954/2009).

\section{COLABORADORES}

CFF MOREIRA participou do planejamento, da coleta, da análise e interpretação dos dados e da elaboração e revisão do artigo. MLM LOPES e VL VALENTE-MESQUITA foram responsáveis pela elaboração do projeto e orientaram o planejamento, a coleta, a análise e interpretação dos dados e a elaboração e revisão do artigo.

\section{REFERÊ NCIAS}

1. Pereira ALF, Vidal TF, Constant PBL. Antioxidantes alimentares: importância química e biológica. Nutrire. 2009; 34(3):231-47.

2. Bianchi MLP, Antunes LMG. Radicais livres e os principais antioxidantes da dieta. Rev Nutr. 1999; 12(2):123-30. doi: 10.1590/S1415-527319990002 00001.

3. Cano A, Medina A, Bermejo A. Bioactive compounds in different citrus varieties. Discrimination among cultivars. J Food Compos Anal. 2008; 21(5):377-81. doi: 10.1016/j.jfca.2008.03.005.

4. Amaro AA, Caser VD. Diversidade do mercado de tangerinas. Informações Econômicas. 2003; 33(12): 51-67.

5. Fennema OR. Química de los alimentos. $4^{\mathrm{a}}$ ed. Porto Alegre: Artmed; 2010.

6. Özkan M, Aysegül K, Cemeroglu B. Effects of hydrogen peroxide on the stability of ascorbic acid during storage in various fruit juices. Food Chem. 2004; 88(4):591-7. doi: 10.1016/j.foodchem.2 004.02.011.

7. Instituto Brasileiro de Geografia e Estatística. Produção agrícola municipal: informações sobre culturas permanentes. Brasília: IBGE; 2010 [acesso 2012 fev 16]. Disponível em: <http://www.sidra. ibge.gov.br/bda/pesquisas/pam/default. asp? $0=18 \& \mathrm{i}=\mathrm{P}>$.

8. Vale AAS, Santos CD, Abreu CMP, Corrêa AD, Santos JA. Alterações químicas, físicas e físico-químicas da tangerina 'ponkan' (Citrus reticulata Blanco) durante o armazenamento refrigerado. Rev Ciên Agrotec. 2006; 30(4):778-86. doi: 10.1590/S1 413-70542006000400027.

9. Rufini JCM, Ramos JD. Influência do raleio manual sobre a qualidade dos frutos de tangerineira 'Ponkan' (Citrus reticulata Blanco). Rev Ciênc Agrotec. 2002; 26(3):516-22. doi: 10.1590/S010029452009000200038.

10. Lima VLAG, Mélo EA, Lima LS. Avaliação da qualidade de suco de laranja industrializado. B CEPPA. 2000; 18(1):95-104.

11. Associação Brasileira das Indústrias de Refrigerantes e de Bebidas não Alcoólicas. Dados de mercado 2011: consumo de todas as bebidas comerciais 
2005-2010. Brasília: ABIR; 2011 [acesso 2012 fev 16]. Disponível em: <http://abir.org.br/tags/ pesquisas-2011/>.

12. Tetra Pack lança campanha para incentivar consumo de suco pronto e água de coco. In: Empreendedor: inovação, gestão e valor aos negócios. 2005 [acesso 2009 mai 4]. Disponível em: <http:// www.empreendedor.com.br/ler.php?cod=703>.

13. Universidade Estadual Paulista. Formas de amostragem [acesso 2009 mar 4]. Disponível em: <ww w.dcce.ibilce.unesp.br/ adriana/engali/Formas deamostragem.pdf>.

14. Instituto Adolfo Lutz. Métodos químicos e físicos para análise de alimentos. In: Normas analíticas do Instituto Adolfo Lutz. $3^{\text {a }}$ ed. São Paulo: IAL; 1985.

15. Association of Official Analytical Chemists. Official methods of analysis. $14^{\text {th }}$ ed. Washington: AOAC; 1984.

16. Benassi MT, Antunes AJ. A comparison of metaphosphoric and oxalic acids as extractants solutions for the determination of vitamin $C$ in selected vegetables. Braz Arch Biol Technol. 1988; 31(4):507-13.

17. Brasil. Agência Nacional de Vigilância Sanitária. Resolução RDC $n^{\circ} 360$, de 23 de dezembro de 2003. Regulamento técnico sobre rotulagem nutricional de alimentos embalados. Diário Oficial da União. 200326 dez; p.33-34, Seção 1.

18. Kuskoski EM, Asuero AG, Morales MT, Fett R. Frutas tropicais silvestres e polpas de frutas congeladas: atividade antioxidante, polifenóis e antocinaninas. Ciênc Rural. 2006; 36(4):1283-7.

19. Brasil. Agência Nacional de Vigilância Sanitária. Resolução RDC n² 269, de 22 de setembro de 2005. Regulamento técnico sobre a ingestão diária recomendada de proteína, vitaminas e minerais. Diário Oficial. 200523 set; p.372, Seção 1.

20. Xu G, Liu D, Chen J, Ye X, Ma Y, Shi J. Juice components and antioxidant capacity of citrus varieties cultivated in China. Food Chem. 2008; 106:545-51. doi: 10.1016/j.foodchem.2007.06.0 46.

21. Damiani C, Boas VBV, Pinto DM. Processamento mínimo de tangerinas armazenadas sob duas temperaturas. Rev Ciênc Agrotec. 2008; 32(1):308-13. doi: $10.1590 / S 1413-70542008000100044$.

22. Lee SK, Kader AA. Preharvest and postharvest factors influencing vitamin $C$ content of horticultural crops. Postharvest Biol Technol. 2000; 20:207-20. doi: 10.1016/S0925-5214(00)00133-2.

23. Lavinas FC, Almeida NC, Miguel MAL, Lopes MLM, Valente-Mesquita VL. Estudo da estabilidade química e microbiológica do suco de caju in natura armazenado em diferentes condições de estocagem. Ciên Tecnol Aliment. 2006; 26(4):875-83. doi: 10.1590/S0101-20612006000400026.

24. Valente-Mesquita VL, Lopes MLM, Sabino GS, Silva PT, Alves BC. Teor de vitamina C em suco de diferentes cultivares de laranja (Citrus sinensis) e em diferentes sucos industrializados. Nutr Bras. 2002; 1(1):34-9.

25. Kabasakalis V, Siopidou D, Moshatou E. Ascorbic acid content of commercial fruit juices and its rate of loss upon storage. Food Chem. 2000; 70(3):3258. doi: 10.1016/50308-8146(00)00093-5.

26. Melo EA, Maciel MIS, Lima VLAG, Nascimento RJ. Capacidade antioxidante em frutas. Rev Bras Ciên Farm. 2008; 44(2):193-201.

27. Klimczak I, Malekca M, Szlachta M, GliszczynskaSwiglo A. Effects of storage on the content of polyphenols, vitamin $\mathrm{C}$ and the antioxidant activity of orange juices. J. Food Compos Anal. 2007; 20(3-4):313-22. doi: 10.1016/j.jfca.2006.02.012.

28. Caleguer VF, Toffoli EC, Benassi MT. Avaliação da aceitação de preparados sólidos comerciais para refresco sabor laranja e correlação com parâmetros físico-químicos. Semina Ciênc Agrar. 2006; 27(4): 587-98.

29. Silva PT, Fialho E, Lopes MLM, Valente-Mesquita VL. Sucos de laranja industrializados e preparados sólidos para refrescos: estabilidade química e físicoquímica. Ciênc Tecnol Aliment. 2005; 25(3):597-602. doi: 10.1590/S0101-20612005000300033.

30. Brasil. Agência Nacional de Vigilância Sanitária. Resolução n 45, de 3 de novembro de 2010. Regulamento técnico sobre aditivos alimentares autorizados segundo as Boas Práticas de Fabricação. Diário Oficial da União. 20105 nov; p.63-68, Seção 1.

31. Universidade Federal de Santa Catarina. Aditivos alimentares [2012 fev 16]. Rev Eletron Dep Quím UFSC [acesso 2012 fev 16]. Disponível em: <http:// www.qmc.ufsc.br/qmcweb/artigos/aditivos.html>.

32. Brasil. Ministério da Saúde. Secretaria de Vigilância Sanitária. Portaria n. 31, de 13 de janeiro de 1998. Regulamento técnico para fixação de identidade e qualidade de alimentos adicionados de nutrientes essenciais. Diário Oficial. 199816 jan; p.4-5, Seção 1.

33. Pereira CQ, Lavinas FC, Lopes MLM, ValenteMesquita VL. Industrialized cashew juices: variation of ascorbic acid and other physicochemical parameters. Ciênc Tecnol Aliment. 2008; 28(Suppl): 266-70. doi: 10.1590/S0101-2061200800050 0040 .
Recebido em: 6/1/2011

Versão final em: 3/7/2012 Aprovado em: 20/8/2012 